This item was submitted to Loughborough's Research Repository by the author.

Items in Figshare are protected by copyright, with all rights reserved, unless otherwise indicated.

\title{
On the quantum-to-classical transition of a particle in a box
}

PLEASE CITE THE PUBLISHED VERSION

https://doi.org/10.1002/andp.201400119

\section{PUBLISHER}

(c) Wiley

\section{VERSION}

AM (Accepted Manuscript)

\section{PUBLISHER STATEMENT}

This work is made available according to the conditions of the Creative Commons Attribution-NonCommercialNoDerivatives 4.0 International (CC BY-NC-ND 4.0) licence. Full details of this licence are available at: https://creativecommons.org/licenses/by-nc-nd/4.0/

\section{LICENCE}

CC BY-NC-ND 4.0

\section{REPOSITORY RECORD}

Paton, Jamie E., Mark J. Wootton, and Mark J. Everitt. 2019. "On the Quantum-to-classical Transition of a Particle in a Box". figshare. https://hdl.handle.net/2134/35169. 


\title{
On the quantum-to-classical transition of a particle in a box
}

\author{
Jamie E Paton ${ }^{1}$, Mark James Wootton ${ }^{2}$, and M.J. Everitt ${ }^{1, *}$
}

The exact formulation of the correspondence principle and in particular understanding the quantum-to-classical transition remains an open problem in quantum mechanics. In this paper we present our investigation into the quantumto-classical transition of the most trivial of quantum systems - a particle in a box. Whilst it is perhaps surprising, even this example can produce new physical insight into these fundamental problems. With modern fabrication techniques of nano-mechanical systems we will be able to experimentally investigate these results and directly observe the quantum-to-classical transition. This will enable us to build technologies that probe the fundamental questions of quantum mechanics, such as the maximum size of a quantum object.

\section{Introduction}

Quantum mechanics is our best theory of reality $[1,2]$. With the exception of gravity, its success in describing our universe is remarkable [3]. The development of quantum 1.0 technologies such as the laser and microchip have already revolutionised the world around us. The emergence of quantum 2.0 technologies $[4,5]$, that directly leverage non-locality and entanglement as core components of their operation, looks set to have a similar disruptive effect. Despite this technological success, the ontological foundations of the subject remain unclear. The same technological sophistication that is enabling new quantum technologies may also provide a route to understanding aspects of these foundational problems. Of particular interest is the correspondence principle, and the emergence of the classical world. In textbook quantum mechanics this is often presented in terms of being achieved by systems in high energy states or in terms of Planck's constant becoming vanishingly small - approaches that are known for being problematic [6]. There has been a lot of work on the correspondence principle that focuses on reproducing chaotic dynamics of classical systems the interest arising because the Schrödinger equation is strictly linear [7-20]. We note that there is a large body of literature that takes a statistical random matrix theory approach to the quantum-to-classical transition in Hamiltonian systems. This is beyond the scope of our dis- cussion and we will focus only on the stronger form of a trajectory-level approach.

Perhaps because on a prima facie level they lack interest, apparently simpler systems, such as a particle bouncing off a barrier, have generally been overlooked in studies on the quantum-to-classical transition. Recently there has been significant progress in this problem [21]. This work does not simply fill a well needed gap in the literature rather it is sophisticated, subtle and opens up some very interesting lines of investigation.

It is well known that when a quantum particle has enough energy to overcome a barrier, the wavefunction splits into transmitted and reflected parts. This is contrary to the classical result where only transmission occurs. Recent work by Halliwell and Bedingham [21] has demonstrated the use of an open quantum system in the suppression of the reflected part. Their work deals with a single encounter with a finite barrier and is an interesting exploration of the quantum to classical transition, the the inherent subtleties, of simple systems. Here we present work that uses a similar approach to the related

\footnotetext{
* Corresponding author E-mail:m.j.everitt@physics.org

1 Department of Physics, Loughborough University, Loughborough, Leicestershire LE11 3TU

2 Department of Mathematics, Loughborough University, Loughborough, Leicestershire LE11 3TU
} 
problem of a particle in a box. The interesting additional element now being that the size of the box also plays a role in achieving the correspondence limit. Specifically we seek to check, for this system, the suitability of the version of the correspondence principle given in [6] that states: "Consider $\hbar$ fixed (it is) and scale the Hamiltonian, in such a way as to preserve the form of the classical phase space, so that when compared with the minimum area $\hbar / 2$ in phase space: 1 . the relative motion of the expectation values of any observable (generalized) co-ordinate (and hence the associated classical action) becomes large, and 2. the state vector is localized (in any representation); then, under these circumstances, expectation values of operators will behave like their classical counterparts.".

In this work we begin to explore how an open systems approach, that can realise the required localisation of the above statement of the correspondence principle, may lead to understanding the quantum-to-classicalcrossover, of such simple systems. The open quantum system dynamics of a particle in a box displays a surprisingly rich set of behaviours where the momentum and mass of the particle, the relative size of the box, and magnitude of environmental decoherence all play a part. We also present an initial study of another interesting area for investigation regarding the quantum-to-classical transition which is provided by the two dimensional Bunimovich stadium - arguably the simplest example of Hamiltonian chaos. This paper explores the question of whether or not an open systems approach will yield a correspondence limit for such trivial Hamiltonian systems. We discuss our findings but a full understanding of the emergence of classical dynamics from these quantum objects is beyond the scope of this paper.

Nano-mechanical realisations of a particle in a box could well be an ideal choice of system for investigating the correspondence principle [22,23]. The question is, 'can we understand the transition from quantum carpets and their revivals to a "particle" bouncing in a box?' There have been a number of works that realise nano-mechanical systems demonstrating quantum effects, which would form natural test systems for this subject (instead of considering analogies, we are now in a position to actually look at real nano-mechanical quantum particles in a box) [22,24-28]. Applications of nanomechanical systems, such as the manipulation of simple quantum devices, have significant implications for future quantum technologies. Recently it has been shown that it is possible to hold and then control objects at nano-scales [29-32]. An example application of characterising the emergence of classical trajectories of nanomechanical systems would be to develop a 'quantumness' test. We could then establish the suitability of a given nano-mechanical device for application in quantum technologies (in a similar fashion to using qubits as a probe to test the 'quantumness' of a field mode [16]).

It is now possible in a laboratory environment to produce nanomechanical objects that exhibit quantum properties [24,33]. Manipulation of these devices will allow us to experimentally explore simple problems such as the one dimensional particle in a box.

\section{Quantum Particle in a Box}

If we consider briefly a classical one-dimensional particlein-a-box. It is easy to see that as the particle simply bounces between the two walls, it would trace out a 'zigzag' shape as it elastically bounces off each wall. As a standard textbook example it is well known that a quantum one-dimensional particle-in-a-box displays markedly different properties to its classical counterpart $[1,2]$. This can be seen by solving the Schrödinger equation for the particle where the initial state is as particle-like as it can be - a coherent state with [34]

$\psi(q)=\left[2 \pi(\Delta \hat{q})^{2}\right]^{-1 / 4} \exp \left[-\left(\frac{q-\langle\hat{q}\rangle}{2(\Delta \hat{q})}\right)^{2}+\frac{i\langle\hat{p}\rangle q}{\hbar}\right]$

In Figure 1 we display one such solution, showing and the expectation value of the position operator $\langle\hat{q}\rangle$. The dynamics of $\langle\hat{q}\rangle$ is shown as a white line in Figure 1 and shares almost nothing in common with the "zig-zag" of its classical counterpart. It is easy to see that if a quantum particle is left in a box and then its position is measured after a random time, there is a much higher chance that it will be found in the middle of the box. This is a measurable difference between the quantum and classical systems - in the classical system every position in the box is equiprobable. This behaviour is well known and we will not discuss it further here. In addition to plotting $\langle\hat{q}\rangle$ we also plot the square of the amplitude of the wave-function, $|\psi(x)|^{2}$. The pattern displayed in this plot is known as a 'quantum carpet' following [35] as the pattern produced by plotting $|\psi(x)|^{2}$ as a function of time is reminiscent of an intricately decorated carpet or rug. It's conventional in quantum carpet plots to plot time along the $y$-axis. A quantum carpet allows one to very easily visually spot the symmetries of the system, and to visually inspect the level of 'quantumness'. We can, for example, see that after a period of time the system starts to "run backwards" until the original Gaussian wavepacket is restored. This is known as the revival of the system - as discussed by Berry et. al. [35] — and is periodic with the period being referred to as the revival time. 


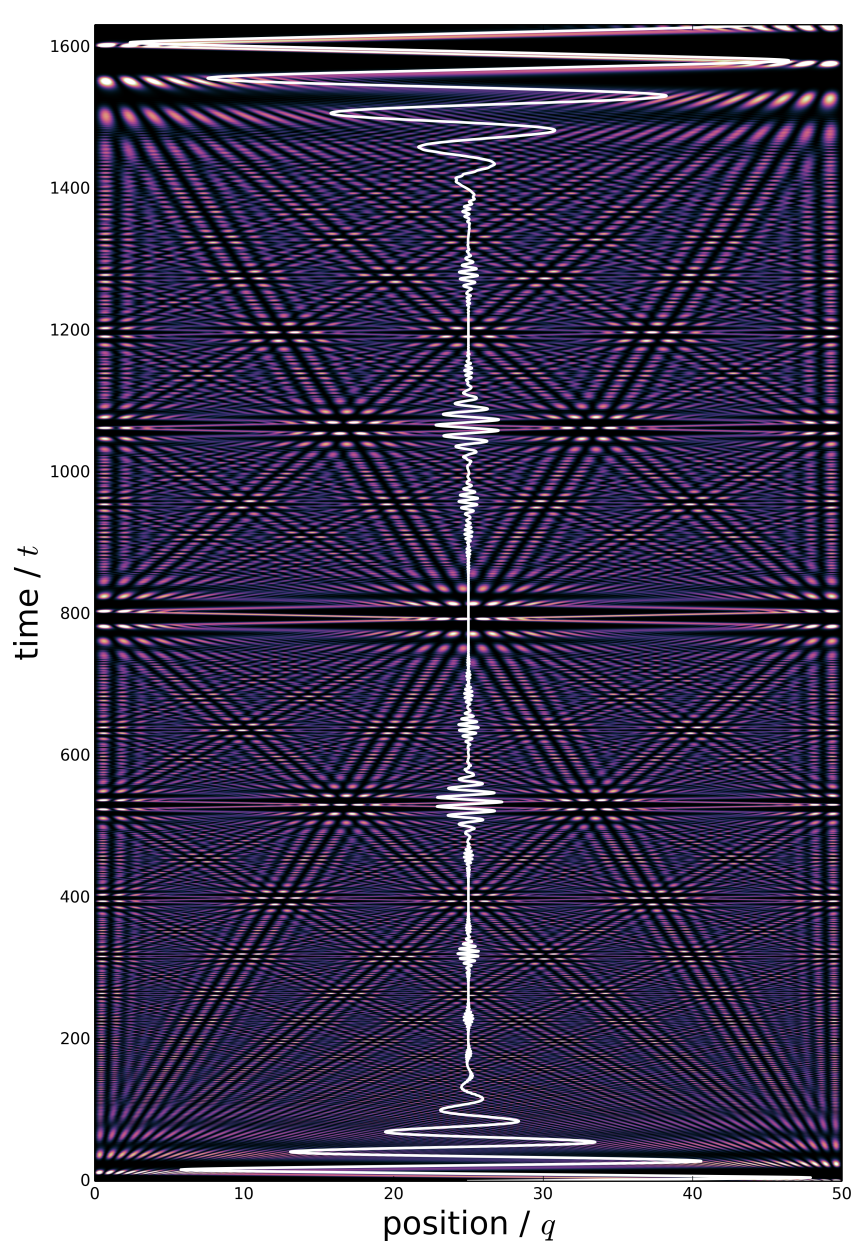

Figure 1 Showing the evolution of $|\psi(x)|^{2}$ for a particle in a 1$D$ infinite potential well where its initial condition is a Gaussian wave-packet with $(\Delta \hat{q})=1$ and $\langle\hat{p}\rangle=2$. The white line plotted is $\langle\hat{q}\rangle$.

Environmental decoherence has been successfully shown to effectively suppress quantum-mechanical reflection [21] and to be a component part of achieving a quantum-to-classical transition in another Hamiltonian system $[13,14]$. We model the effects of environmental decoherence by using quantum state diffusion (QSD). This takes the form of a stochastic Itô increment equation that is a single unravelling of the Linblad master equation

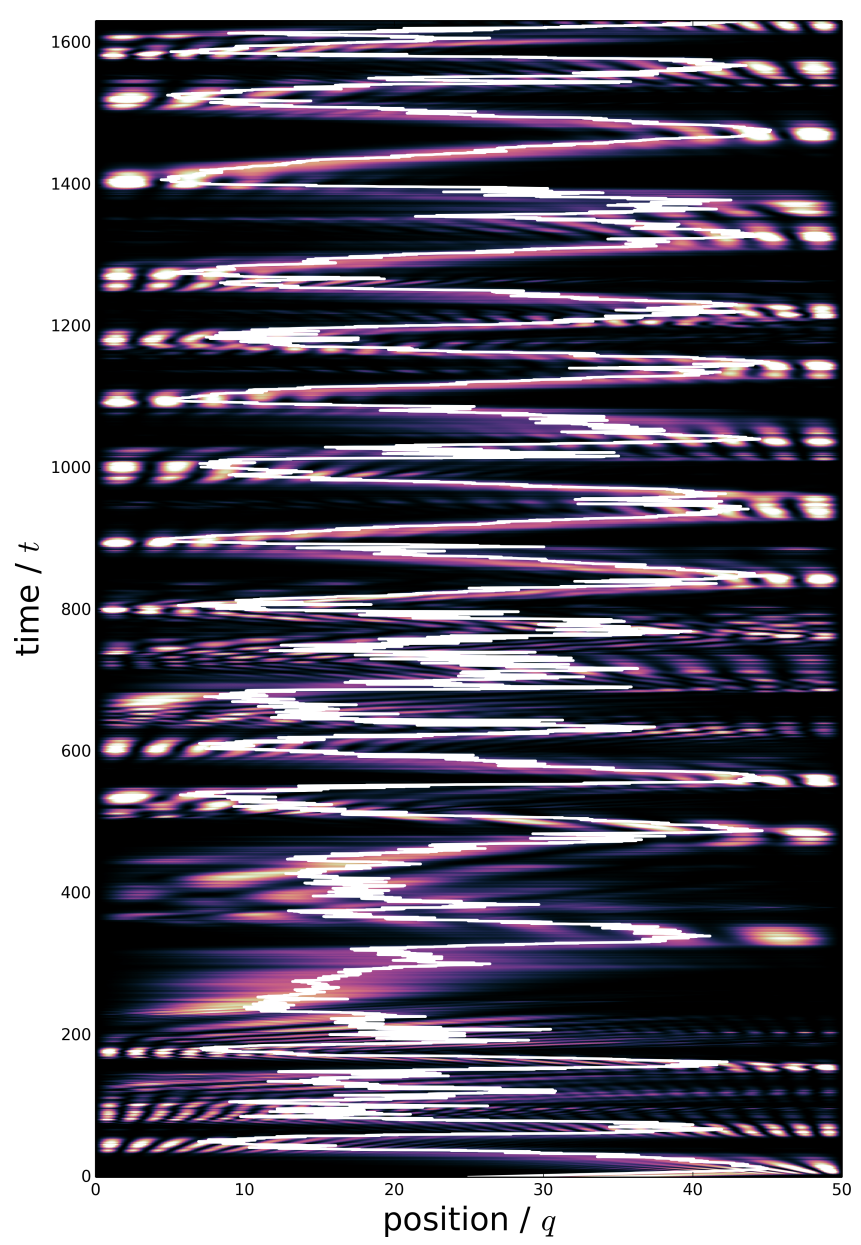

Figure 2 Showing the evolution of $|\psi(x)|^{2}$ for a particle in a 1$D$ infinite potential well subject to environmental decoherence using the quantum state diffusion unravelling of the master equation. The initial condition is a Gaussian wave-packet with $(\Delta \hat{q})=1,\langle\hat{p}\rangle=2$ and the decoherence factor is $\hat{L}_{1}=0.01 \hat{q}$. The white line plotted is $\langle\hat{q}\rangle$. Here we see the emergence of behaviour that is more like that of a classical particle bouncing around in a box, including localisation of the wave-packet, rather than quantum-coherent carpets of the equivalent closed quantum system.

given by

$$
\begin{aligned}
\mathrm{d}|\psi\rangle & =-\frac{i}{\hbar} \hat{H}|\psi\rangle \mathrm{d} t \\
& +\sum_{j}\left[\left\langle\hat{L}_{j}^{\dagger}\right\rangle \hat{L}_{j}-\frac{1}{2} \hat{L}_{j}^{\dagger} \hat{L}_{j}-\frac{1}{2}\left\langle\hat{L}_{j}^{\dagger}\right\rangle\left\langle\hat{L}_{j}\right\rangle\right]|\psi\rangle \mathrm{d} t \\
& +\sum_{j}\left(\hat{L}_{j}-\left\langle\hat{L}_{j}\right\rangle\right)|\psi\rangle \mathrm{d} \xi_{j}
\end{aligned}
$$




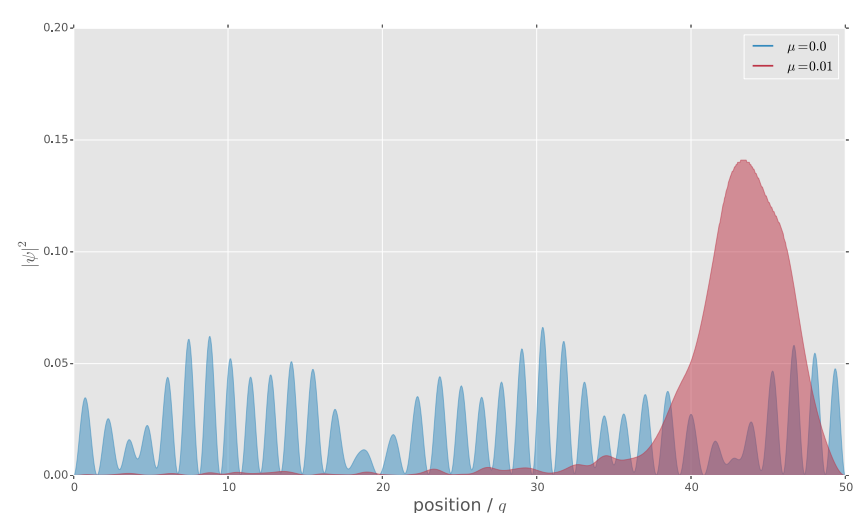

Figure 3 Cross-section of the amplitude of the quantum state at dimensionless time $t \approx 980$ of Figures 1 and 2 showing localisation of the wave-packet for the example with decoherence.

Here the first term is regular Schrödinger evolution, the second term models the drift of the wavefunction, and the third term models the stochastic fluctuation effects. The $\mathrm{d} \xi_{j}$ is a random complex Gaussian obeying $\overline{\mathrm{d} \xi_{j}}=0$, $\overline{\mathrm{d} \xi_{j} \mathrm{~d} \xi_{j}^{\dagger}}=0$ and $\overline{\left|\mathrm{d} \xi_{j}\right|}=\mathrm{d} t$. These conditions ensure that the random complex Gaussians are independent, not covariant (their real and imaginary parts are independent), and that they are normalised [36]. QSD models continuous measurement of the wavefunction [36] as a Markov process, that is to say that the environment is sufficiently large enough to have no memory, hence the independent stochastic effects. For this example we choose, due to its success in achieving a correspondence limit for another conservative system $[13,14]$, to have the one Linblad operator $\hat{L}_{1}=0.01 \hat{q}$. We note that the numerical modelling of equation (2) for the system under consideration is not well suited to traditional methods of solving non-linear differential equations such as the split step Fourier method or Heisenberg matrix representations. Our computations were therefore conducted using a much slower finite volume method (FVM) of reference [37]. This has placed some limitations of the computations that we were able to perform.

In Figure 2 we show the dynamics of a particle in a 1-D infinite potential well subject to environmental decoherence modelled using quantum state diffusion. Here we see the emergence of behaviour that is more like that of localised particle bouncing around in a box rather than quantum-coherent carpets of the equivalent closed quantum system. To more clearly show this environmentally induced localisation we show in Figure 3 the amplitude of the quantum state at dimensionless time $t \approx 980$ of Figures 1 and 2. Compared to the classical case of stochastic

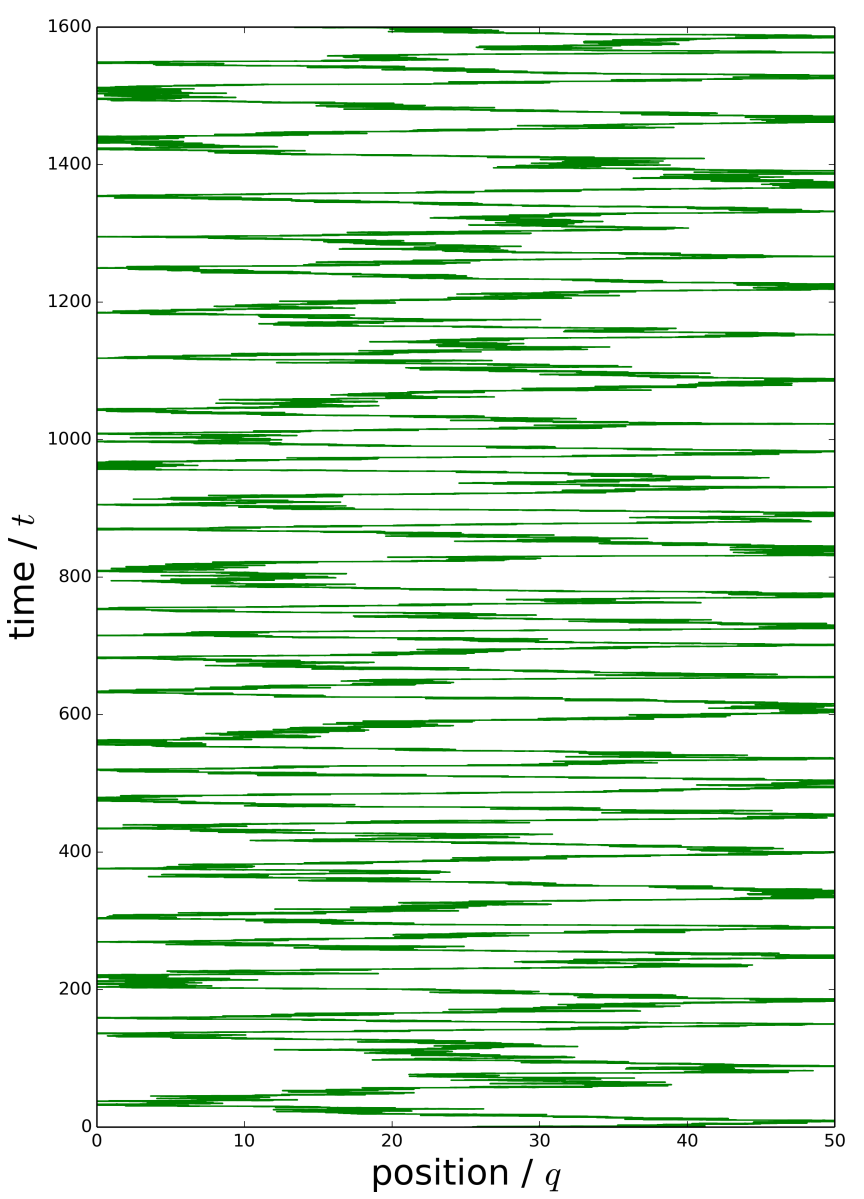

Figure 4 Showing the evolution of position for a classical particle in a 1-D infinite potential well with environmental effects (Brownian motion) [36].

environmental effects (Brownian motion) shown in Figure 4 , the evolution of $\langle\hat{q}\rangle$ is remarkably similar. Note that a classical Brownian motion would arise from thermal fluctuations and our current model assumes a zero temperature. Figure 4 is therefore presented for qualitative comparison. The addition of a thermal bath to our model will be the subject of future work. The convergence is not sufficiently good for us to be able to strongly claim that we have achieved the correspondence limit. There are several reasons for this. Firstly the box is only about an order of magnitude or so larger than the wave-packet/Planck cell. Indeed the box is sufficiently small that, in contrast to the classical dynamics, the expectation value of position always remains noticeably away from the box's boundary. Previous studies have observed that a large action is needed and our simulations fall a fraction short of meeting this requirement [6]. Secondly, we note that the initial momentum (and therefore energy) of this particle is small. 


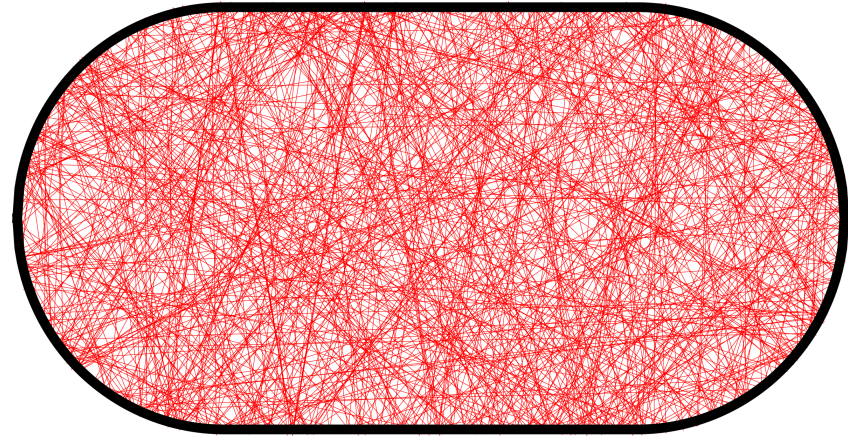

Figure 5 Configuration space for a classical particle in the Bunimovich stadium showing a typical example of Hamiltonian chaos. Here the initial momenta were $p_{x}=1$ and $p_{y}=0.6$ and the particle was initially in the centre of the stadium.

The noise from environmental fluctuations therefore has a significant influence on the particle's dynamics (as would be the case for a classical particle subject to significant Brownian motion). Previous studies of another Hamiltonian system, namely the three body problem $[13,14]$, indicate that provided the initial energy is large enough it should be possible to recover a quantum-to-classical transition that can be, to good approximation, considered to be conservative. The same conditions need to be met for the particle in a one dimensional box. To achieve its correspondence limit, the computations, requiring larger boxes and initial momentum, have proved surprisingly demanding and will be the subject of future work.

Caveats aside, our model is sufficiently good to show (i) particle-like localisation of the wave-packet (ii) suppression of quantum revivals and (iii) very good qualitative agreement between $\langle\hat{q}\rangle$ and the classical dynamics of a particle-in-a-box subjected to a Brownian motion. For these reasons we believe that we have demonstrated a possible mechanism for the quantum to classical crossover of a particle in a one-dimensional box.

In understanding the quantum-to-classical transition the emergence of classical chaos is often taken as a signature of achieving the correspondence limit [7-20]. Such a test is not possible for a particle in a one dimensional box. However, in two dimensions such an investigation should be achievable through the use of boxes with certain boundary conditions such as the Bunimovich stadium $[38,39]$. In our view it should be possible to realise nano-mechanical devices of this kind which should form an ideal platform to test their quantumness. In the correspondence limit we expect trajectories that look like those of a classical particle, together with exponential divergence of any two trajectories - very different be- (a) Schrödinger Evolution

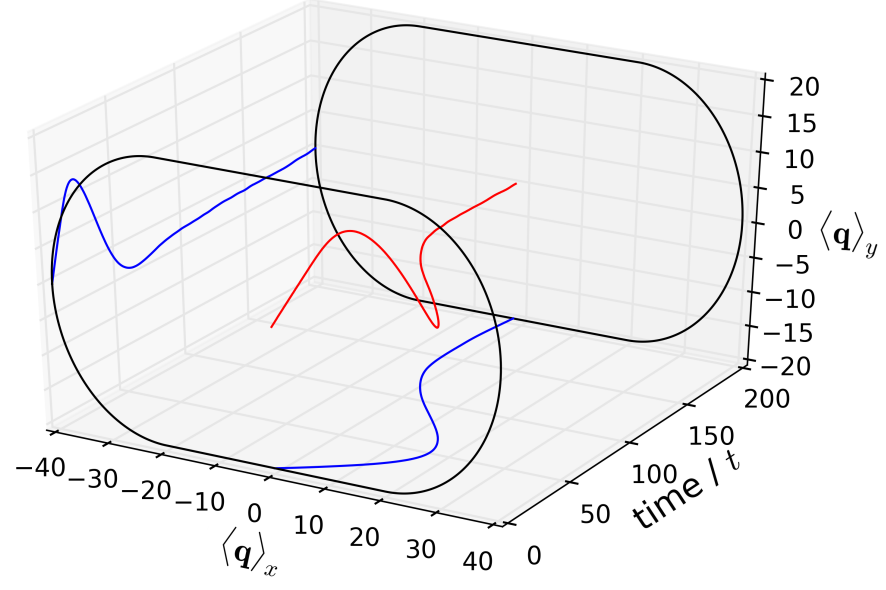

(b) Quantum State Diffusion

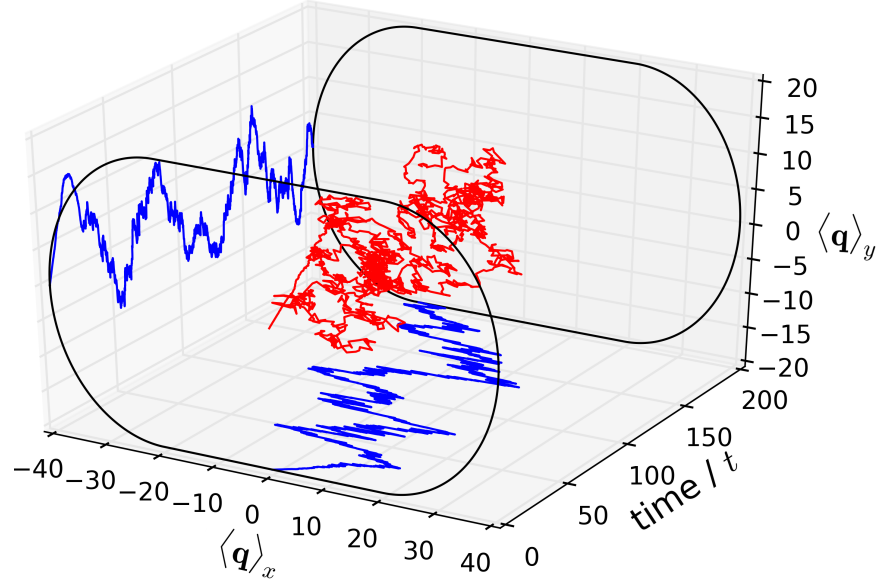

Figure 6 Showing the evolution of $\left(\langle\hat{q}\rangle_{x},\langle\hat{q}\rangle_{y}\right)$ for a quantum particle in the Bunimovich stadium with initial condition of a Gaussian wave-packet centred in the stadium with $\langle\hat{p}\rangle_{x}=$ 0.6 and $\langle\hat{p}\rangle_{y}=1$ for (a) regular Schrödinger evolution and (b) subject to environmental decoherence using the quantum state diffusion with $\hat{L}_{1}=0.01 \hat{q}_{x}$ and $\hat{L}_{2}=0.01 \hat{q}_{y}$. Here (a) is consistent with the known phenomena of the amplitude of the wave-packet spreading out to cover the entire configuration space whilst in (b) there are the beginnings of the development of classical like trajectories, albeit strongly influenced by noise, and the dynamics are manifestly different from the quantum coherent evolution.

haviour from the quantum limit. The Bunimovich stadium has been the subject of numerous investigations of weak forms of the correspondence principle based on a 
statistical random matrix theory approach that we will not discuss here as we are interested in the stronger trajectory level - realisations of the quantum to classical transition. In Figure 5 we show the configuration space for an example of a classical particle in a Bunimovich stadium exhibiting Hamiltonian chaos. In Figure 6 we display the evolution of $\left(\langle\hat{q}\rangle_{x},\langle\hat{q}\rangle_{y}\right)$ for a quantum particle in the Bunimovich stadium. Initially the quantum state is a Gaussian wave-packet centred in the stadium with $\langle\hat{p}\rangle_{x}=0.6$ and $\langle\hat{p}\rangle_{y}=1$. In Figure 6(a) we consider Schrödinger evolution and the dynamics of $\langle\hat{q}\rangle_{x}$ and $\langle\hat{q}\rangle_{y}$. This shows collapse-like behaviour similar to that seen in $\langle\hat{q}\rangle$ for the one-dimensional case. Such behaviour is typical and can clearly be seen to be very different from the dynamics of a classical particle in the same stadium. We might therefore ask if a similar open systems approach, that has often proved to be effective, could be used to realise the correspondence limit of the Bunimovich stadium at a trajectories level.

Once more we can make use of quantum state diffusion to model environmental decoherence, now using the two Lindblad operators $\hat{L}_{1}=0.01 \hat{q}_{x}$ and $\hat{L}_{2}=0.01 \hat{q}_{y}$. While the noise is a strong influence, bouncing is evident and the dynamics are manifestly different from the quantum coherent evolution. The emergence of a classical correspondence is supported by (i) $\langle\hat{q}\rangle$ not drifting towards the centre of the stadium and staying there, i.e. there is no collapse and there are noticeable bounces (ii) although not shown - that the environment induces a localisation of the wave function to a particle-like Gaussian. Hence, as in the one-dimensional case we can see the beginnings of the development of classical-like trajectories. In order to more fully explore this quantum to classical crossover we will conduct further investigations that will make use of larger stadia and initial states with greater momenta which we will report on in due course. With the above caveats in place, the open systems approach looks to be a successful route to realising the quantum to classical transition and should recover chaos from the simplest of classically chaotic Hamiltonian systems.

\section{Conclusion}

We have studied the quantum-to-classical transition of a particle in a one-dimensional-box using an quantum state diffusion approach. Because of its apparent triviality this system has until now been overlooked (as discussed in the introduction, there have been many studies on "more interesting" systems). Our work is related to a recent study of the correspondence limit of particle sub- ject to a finite barrier [21]. Here we observe that when considering the quantum-to-classical transition of particles in a box, the of size of box (which needs to be large relative to the action) should also be taken into account. This is something that does not come across from the study of a single barrier. We have also presented the first study of a quantum trajectories approach to a particle in a Bunimovich stadium (up until now studies of this system have taken a random matrix theory approach). We have observed the apparent beginnings of a quantum-toclassical transition. Specifically, we see localisation of the wave-packet and bouncing between the walls. More work needs to be done in order to conclude that an arbitrarily good correspondence limit can be achieved and recover classical-like Hamiltonian chaotic dynamics, however the key ingredients are all present and our prescription looks to be a successful one. It would be interesting to explore the possibility of extracting the measurement record from the environmental decoherence. We have suggested, in line with [22], that nano-mechanical systems would be a good and appropriate platform for testing the quantumto-classical transition. Observing the quantum to classical transition may be possible, following [16], using qubits as a "quantumness" probe and an experimental setup similar to that found in [40] where a nano-mechanical device was used to measure a superconducting qubit (but now reversing the role of the mechanical system and the qubit).

\section{Acknowledgements}

MJE would like to thank Michael Berry and John Samson for interesting and informative discussions. Quantum simulation plots used a specific cubehelix colourmap provided by [41].

Key words. Particle in a box, Nano-mechanics, quantum to classical transition, correspondence principle

\section{References}

[1] C. Cohen-Tannoudji, B. Diu, and F. Laloë, Quantum mechanics, Quantum Mechanics (Wiley, 1977).

[2] R. P. Feynman, R. B. Leighton, and M. Sands, The Feynman Lectures on Physics (Basic Books, 2010).

[3] R. Feynman, Q E D:, Penguin Books (Penguin Books, Limited, 1990).

[4] J. Pritchard and S. Till Defence Science and Technology Laboratory (2014). 
[5] H. Wiseman and G. Milburn, Quantum Measurement and Control (Cambridge University Press, 2009).

[6] M. J. Everitt New Journal Of Physics 11(1), 013014 (2009).

[7] T. P. Spiller and J. F. Ralph, Phys. Lett. A 194, 235 (1994).

[8] T. Spiller, T. Clark, H. Prance, R. Prance, and J. Ralph J. Low Temp. Phys. 101, 279-303 (1995).

[9] T. A. Brun, N. Gisin, P. F. O’Mahony, and M. Rigo, Phys. Lett. A 229, 267 (1997).

[10] T. Brun, I. Percival, and R. Schack, J. Phys. A 29, 2077 (1996).

[11] W. H. Zurek, Rev. Mod. Phys. 75, 715-775 (2003).

[12] R. Schack, T. Brun, and I. Percival, J. Phys. A 28, 5401 (1995).

[13] M. Everitt Phys. Rev. E 75, 036217 (2007).

[14] N. Burić Physics Letters A 374(40), 4090 - 4098 (2010).

[15] B. Greenbaum, S. Habib, K. Shizume, and S. B. Phys. Rev. E 76, 046215 (2007).

[16] M. J. Everitt, W. J. Munro, and T. P. Spiller Phys. Rev. A 79(Mar), 032328 (2009).

[17] M. Giannoni, A. Voros, and J. Zinn-Justin Elsevier Science(Oct) (1989).

[18] T. Dittrich and R. Graham Ann Phys-New York 200(2), 363-421 (1990).

[19] G. Carlo, G. Benenti, G. Casati, and D. Shepelyansky Phys. Rev. Lett. 94(16) (2005).

[20] S. Habib, K. Jacobs, and K. Shizume Phys. Rev. Lett. 96(1) (2006).

[21] D. J. Bedingham and J. J. Halliwell Phys. Rev. A 88(Aug), 022128 (2013).

[22] A. Asadian, C. Brukner, and P. Rabl Phys. Rev. Lett. 112(May), 190402 (2014).

[23] P. Mohanty Applications of Nonlinear Dynamics (2009).

[24] A. D. O'Connell, M. Hofheinz, M. Ansmann, R. C. Bialczak, M. Lenander, E. Lucero, M. Neeley, D. Sank, H. Wang, M. Weides, J. Wenner, J. M. Martinis, and A. N. Cleland Nature 464(7289), 697-703 (2010).

[25] K. Stannigel, P. Komar, S. J. M. Habraken, S. D. Bennett, M. D. Lukin, P. Zoller, and P. Rabl Phys. Rev. Lett. 109(Jul), 013603 (2012).

[26] A. A. Clerk, F. Marquardt, and J. G. E. Harris Phys. Rev. Lett. 104(May), 213603 (2010).

[27] M. A. Castellanos-Beltran, D. Q. Ngo, W. E. Shanks, A. B. Jayich, and J. G. E. Harris Phys. Rev. Lett. 110 (Apr), 156801 (2013).

[28] P. Rabl, S. Kolkowitz, F. Koppens, J. Harris, P. Zoller, and M. Lukin Nature Physics 6(8), 602-608 (2010).

[29] R. S. A. of Sciences, Measuring and manipulating individual quantum systems, 2012.

[30] M. Dienerowitz, M. Mazilu, and K. Dholakia Journal of Nanophotonics 2(1), 021875-021875-32 (2008).

[31] H. Zheng Nanoscale 5, 4070-4078 (2013).
[32] M. Dienerowitz, M. Mazilu, P. J. Reece, T. F. Krauss, and K. Dholakia Opt. Express 16(7), 4991-4999 (2008).

[33] A. Gaidarzhy, G. Zolfagharkhani, R. L. Badzey, and P. Mohanty Phys. Rev. Lett. 94(Jan), 030402 (2005).

[34] E. Merzbacher, Quantum Mechanics, 3rd edition (John Wiley \& Sons, Inc., New York, 1998).

[35] M. Berry, I. Marzoli, and W. Schleich Physics World (2001).

[36] I. Percival, Quantum State Diffusion (Cambridge University Press, 1998).

[37] J. E. Guyer, D. Wheeler, and J.A. Warren Computing in Science and Engineering 11 (3), 6-15 (2009).

[38] L. Bunimovich Functional Analysis and Its Applications 8(3), 254-255 (1974).

[39] L. Bunimovich Communications in Mathematical Physics 65(3), 295-312 (1979).

[40] M. D. LaHaye, J. Suh, P. M. Echternach, K. C. Schwab, and M. L. Roukes Nature 459(7249), 960-964.

[41] J. Davenport, Cubehelix colormap for python, 2014. 